\title{
Sleep supports inhibitory operant conditioning memory in Aplysia
}

\author{
Albrecht P. A. Vorster ${ }^{1,2}$ and Jan Born ${ }^{1}$ \\ ${ }^{1}$ Institute of Medical Psychology and Behavioral Neurobiology and Center for Integrative Neuroscience (CIN), University of Tübingen, \\ 72076 Tübingen, Germany; ${ }^{2}$ Graduate Training Centre of Neuroscience (GTC)/International Max Planck Research School (IMPRS) at \\ the University of Tübingen, 72076 Tübingen, Germany
}

\begin{abstract}
Sleep supports memory consolidation as shown in mammals and invertebrates such as bees and Drosophila. Here, we show that sleep's memory function is preserved in Aplysia californica with an even simpler nervous system. Animals performed on an inhibitory conditioning task ("learning that a food is inedible") three times, at Training, Retrieval 1, and Retrieval 2, with 17-h intervals between tests. Compared with Wake animals, remaining awake between Training and Retrieval 1, Sleep animals with undisturbed post-training sleep, performed significantly better at Retrieval 1 and 2 . Control experiments testing retrieval only after $\sim 34 \mathrm{~h}$, confirmed the consolidating effect of sleep occurring within $17 \mathrm{~h}$ after training.
\end{abstract}

[Supplemental material is available for this article.]

Sleep is thought to serve the consolidation of memory, and this concept has been scrutinized mainly in mammals (Watson and Buszaki 2015, Westermann et al. 2015, Vorster and Born 2015). If this memory function of sleep represents a fundamental useful adaptation, it might be conserved during evolution. Specifically, as signs of sleep are present in all animals carefully studied so far (Campbell and Tobler 1984; Cirelli and Tononi 2008), we might ask whether the memory function of sleep is analogously present in organisms that are evolutionary rather distant from mammals. Indeed, there is evidence for a supportive role of sleep in memory formation in invertebrates. In Drosophila, inhibitory conditioning of courtship behavior which is a relatively more complex form of learning, robustly benefits from sleep (Ganguly-Fitzgerald et al. 2006; Donlea et al. 2011), whereas effects of sleep are less consistent with simpler types of learning, such as classical conditioning (Le Glou et al. 2012). Similarly, in honeybees, sleep enhanced odor-associated contextual and extinction memories, whereas simple classical conditioning of odor-sucrose reward associations remained unchanged (Hussaini et al. 2009, Zwaka et al. 2015). Also, sleep enhanced the path finding ("homing") of bees (Beyaert et al. 2012).

Aplysia stands out as a model organism with a rather small number of neurons in a distributed nervous system, and is thus a good candidate to test the fundamentality of sleep's memory function and its neural basis. Drosophila and bees, in contrast, possess a centralized brain composed of 10 times more neurons. These features of the Aplysia nervous system allowed deciphering the basics of learning in Aplysia (Kandel et al. 2014). Previous experiments showed that rest states in Aplysia californica which occur predominantly at night in these diurnal animals, fulfill all crucial criteria of sleep (Vorster et al. 2014). We here asked whether sleep in Aplysia impacts consolidation of memory in an inhibitory operant conditioning task, i.e., learning that food is inedible (LFI) (Botzer et al. 1998; Katzoff et al. 2002; Michel et al. 2011, 2012).

Corresponding author: jan.born@uni-tuebingen.de

Article is online at http://www.learnmem.org/cgi/doi/10.1101//m.045054. 117. Freely available online through the Learning \& Memory Open Access option.
Each of 89 Aplysia californica (130-250 g; South Coast Bio-Marine, San Pedro, CA), entrained to a $12 \mathrm{~h} / 12 \mathrm{~h}$ light-dark cycle (lights on at 8:00 h) performed on the LFI task on three occasions, (i) Training phase, (ii) Retrieval 1, and (iii) Retrieval 2, with the testing occasions separated by retention intervals of $\sim 17 \mathrm{~h}$ (Fig. 1, for detailed methods see Supplemental Material). A 17-h interval was chosen based on previous and own studies indicating that signs of long-term memory on the LFI task are not present $12 \mathrm{~h}$ after training, and develop until $24 \mathrm{~h}$ after training (Michel et al. 2012). The Sleep group of animals $(n=31)$ was trained in the evening between 18:00 and 20:00 $\mathrm{h}$, with Retrieval 1 taking place the next day between 11:00 and 13:00 $\mathrm{h}$, after a retention interval including uninterrupted nocturnal rest. Retrieval 2 took place between 4:00 and 8:00 h. The Wake group $(n=19)$ was trained between 8:00 and 10:00 h, with Retrieval 1 taking place between 1:00 and 3:00 $\mathrm{h}$ at night after a retention period of continuous wakefulness supported, if necessary, by sleep deprivation. Retrieval 2 took place at the end of the following light phase between 18:00 and 22:00 h, with this period containing undisturbed rest. Sleep deprivation after training was established by gentle handling (slightly displacing the animal with a plastic ruler) introduced whenever the animal did not show any activity for more than $1 \mathrm{~min}$. It was rarely necessary during the light period $(\sim 2$ stimulations $/ \mathrm{h})$ and more frequent after lights off ( $\sim 4$ stimulations/h). Animals of the sleep group showing $<250$ min of post-training sleep $(n=6)$ and animals of the wake group showing $>200$ min sleep after training $(n=4)$ were also excluded from analysis.

On the LFI task, the animals are presented with inedible food: a $2.5 \times 4 \mathrm{~cm}$ piece of Ulva lactuca algae wrapped in a plastic net of 1-mm spacing held by a hemostat (Fig. 1A). Animals bite and try to swallow the bag containing the algae, which is impossible. In response, the bag is spit out by the animal but held in contact to the lips by the experimenter until the next swallowing response (bite) is elicited. Training stopped if the animal did not swallow

(C) 2017 Vorster and Born This article, published in Learning \& Memory, is available under a Creative Commons License (Attribution-NonCommercial 4.0 International), as described at http://creativecommons.org/licenses/by$\mathrm{nc} / 4.0 /$. 
A

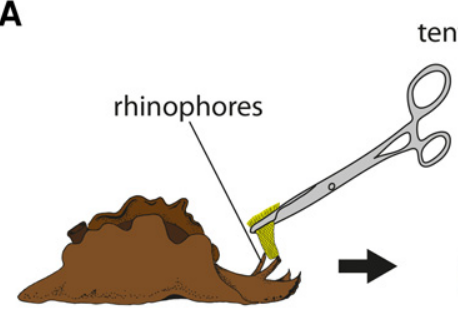
tentacles

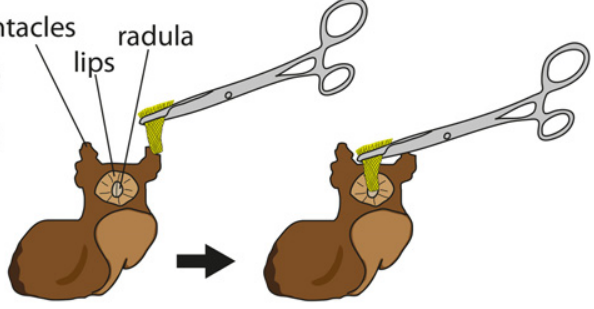

B

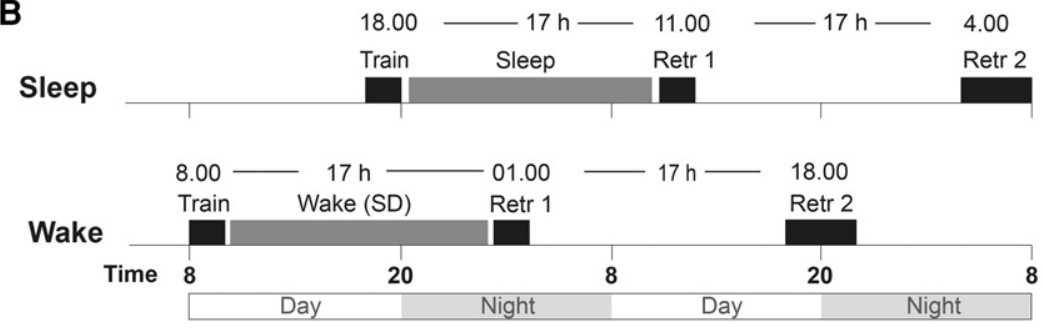

Figure 1. Experimental task and study design. (A) Task "learning that a food is inedible" (LFI). During training the experimenter touches initially the rhinophores and then the tentacles of the Aplysia with a piece of netted Ulva algae. In response the animal orients to the food source, bites, tries to swallow the netted algae, which, however, is impossible, and eventually spits it out again. Training consists of repeated bites and spitting of the food bag, until the animal does not swallow the bag for more than $180 \mathrm{sec}$ or until a maximum of $30 \mathrm{~min}$ of continuous training is reached. Memory expresses itself as a faster relearning, i.e., as a decrease in total response time (first bite to last spitting), and in total time the bag is kept in the mouth (total mouth time). (B) Design. A Sleep group and a Wake group of animals was tested on three occasions, Training, Retrieval 1 (Retr 1), and Retrieval 2 (Retr 2) which used identical procedures, and were separated by $\sim 17-\mathrm{h}$ retention intervals. Training took place in the Sleep group between 18:00 and 20:00 h (i.e., before the animal's natural rest phase) and in the Wake group between 8:00 and 10:00 $\mathrm{h}$ (i.e., at the beginning of the active phase). The experimental 17-h Sleep and Wake phases are indicated by horizontal gray bars.

the netted Ulva for more than $180 \mathrm{sec}$, or after a total response time of continuous biting and swallowing events of $30 \mathrm{~min}$. Task procedures were identical for the Training and Retrieval phases. Performance was measured by the accumulated time the food was kept in the mouth cavity (total mouth time) and the total time the animal engaged in biting and spitting the food (total response time). Animals with $<200 \mathrm{sec}$ of accumulated mouth time or a total response time $<600 \mathrm{sec}$ during Training were excluded from analysis $(n=4)$, as previous studies indicated that these animals show unreliable learning outcome, and to homogenize the groups with regard to training performance (Levitan et al. 2012). Results were analyzed using analysis of variance including a group factor representing the Sleep and Wake groups and a repeated-measures factor representing the different test occasions (Training, Retrieval 1, and Retrieval 2). Post hoc $t$-tests were performed to specify significant main and interaction effects. A $P$-value $<0.05$ was considered significant.

Both the Sleep and Wake groups showed memory for the LFI task at Retrieval 1 in terms of a significantly reduced total response time and total mouth time, in comparison with performance at the Training phase $\left(F_{(1,48)}>71, P<0.0001\right.$, for respective main effects of Training/Retrieval 1, see Fig. 2, for results of post hoc $t$-tests). Whereas others (e.g., Botzer et al. 1998) showed an expression of long-term memory on the LFI task after $24 \mathrm{~h}$, this result indicates that respective long-term memory is formed already substantially earlier, i.e., within $17 \mathrm{~h}$ after training. Importantly however, the gain in performance across the 17 -h retention interval, both in terms of reductions in total mouth time and total response time, was distinctly greater for the Sleep than the Wake group $\left(F_{(1,48)}=5.43, P=0.024\right.$ and 5.40, $P=0.024$, for respective Sleep/Wake $\times$ Training/Retrieval interaction). Memory formation in the Sleep group was rather robust with all but one animal substantially improving performance at Retrieval 1, whereas performance in the Wake group was more heterogeneous with 5 of the 19 animals showing little improvement $(<30 \%)$ or even slightly diminished performance at Retrieval 1 in comparison with the Training phase $(F=3.66, \quad P=0.002$ and $F=2.399$, $P=0.033$, for between-groups difference in variance in total mouth time and total response time, respectively between Sleep and Wake groups at Retrieval 1, Fig. 2). Performance during Training was comparable between the Sleep and Wake groups for both total mouth time $(P>0.28)$ and total response time $(P>0.74$; see Supplemental Material for further analyses).

Retrieval 2 was introduced to test whether a subsequent period of undisturbed sleep would compensate for the poorer memory performance at Retrieval 1 in the Wake group. Both Sleep and Wake groups showed comparable sleep between Retrieval 1 and 2 (see below), and both groups also displayed comparable improvements in total mouth time and total response time across this second interval $\left(F_{(1,39)}>5,73 \quad P<0.031\right.$, for respective Sleep/Wake main effects, $P>0.8$ for respective Sleep/Wake $\times$ Retrieval $1 / 2$ interactions). Accordingly, performance at Retrieval 2 in the Sleep group remained superior to that of the Wake group in terms of total response time $(P=0.011)$ and total mouth time ( $P=0.010$, see Fig. 2 for pairwise statistical comparisons). Thus, sleep occurring after the first 17 post-training hours did not compensate for the memory deficit resulting from wakefulness during the initial $17-\mathrm{h}$ post-training period.

Analyses of sleep-like states (defined by intervals of inactivity $>1 \mathrm{~min}$ ) confirmed that in the Wake group sleep was reduced during the 17 -h post-training interval to on average $87 \pm 53 \mathrm{~min}$, whereas the Sleep group spent on average $493 \pm 116 \mathrm{~min}$ in a sleep-like state during this time $(P<0.0001$, Fig. 2C). During the second 17-h interval between Retrieval 1 and 2, the Wake group showed again normal amounts of sleep-like behavior, which were not significantly different from the Sleep group $(P>$ 0.1 ), with no clear signs of rebound sleep. In the Sleep group, the time spent in a sleep-like state during the Training-toRetrieval 1 interval was correlated with the improvement in LFI performance across this interval $(r=-0.589, P<0.0001$ and $r=-0.392, P=0.024$, for the decrease in total mouth time and total response time, respectively, Fig. 2D). Similar correlations were obtained across the second Retrieval 1-to-Retrival 2 interval ( $r=-0.465, P=0.029$ and $r=-0.436, P=0.043$, respectively, Fig. 2E). Altogether these findings indicate that whenever substantial performance gains occurred across retention intervals covering sleep, these were linked to the time the animal spent in a sleep-like state.

The Wake group displaying inferior performance levels after the 17-h wake interval at Retrieval 1 did not recover performance levels comparable to that of the Sleep group at Retrieval 2, but remained at relatively inferior levels although they exhibited normal sleep-like behavior prior to Retrieval 2. This pattern argues in favor of an effect of sleep on consolidation processes active during the post-training interval, and against an effect of sleep 
A

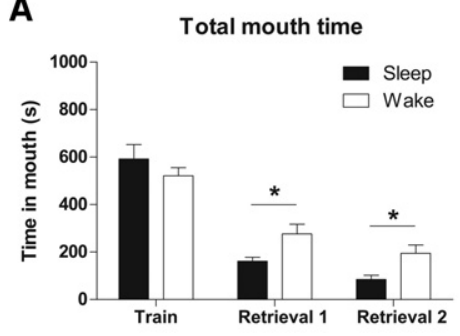

B
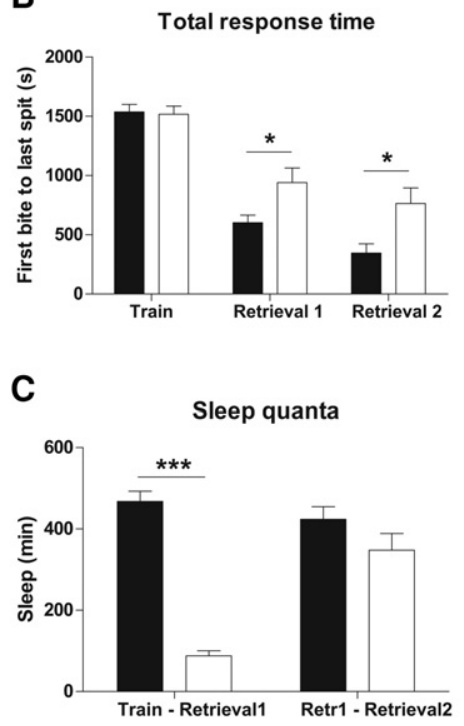

E

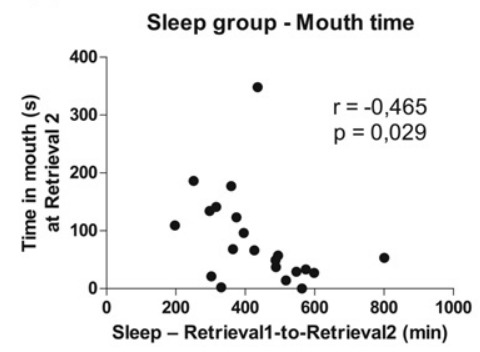

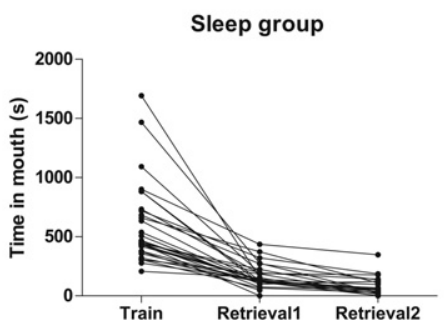

Sleep group
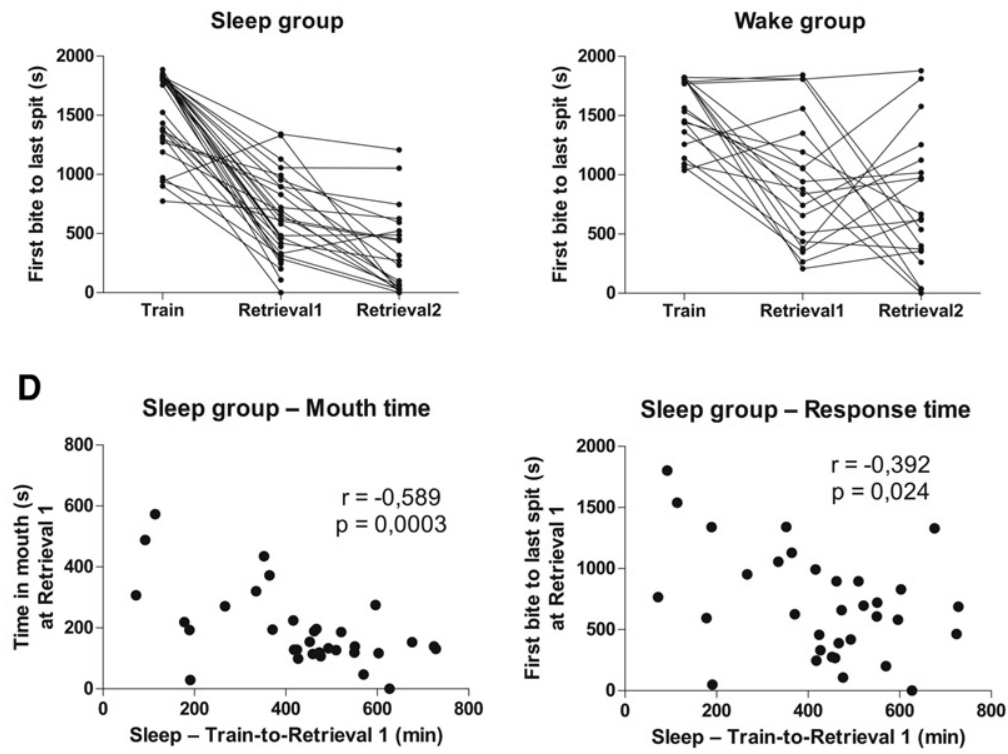

$\mathbf{F}$

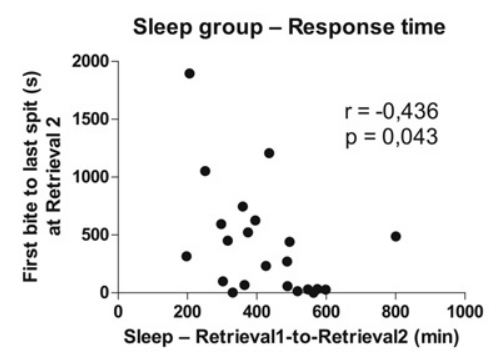

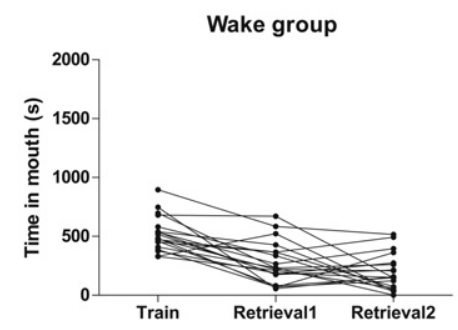
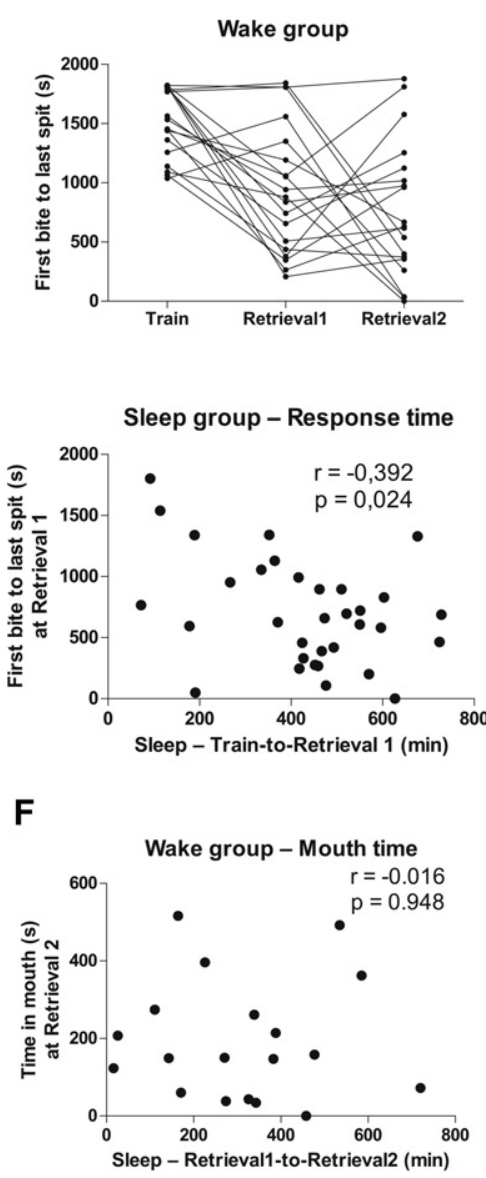

Figure 2. Aplysia show better memory when Training is followed by a 17-h retention interval covering uninterrupted sleep. Animals of the Sleep group (black bars in left panels, $n=31$ ) showed a stronger decrease in $(A)$ total mouth time and $(B)$ total response time at Retrieval 1, with reference to Training, than animals of the Wake group (white bars, $n=19$ ), with this difference between Sleep and Wake groups being maintained at Retrieval 2. Middle and right panels plot individual task performance of animals in both groups. Means $\left( \pm\right.$ SEM), are indicated, $\left({ }^{*}\right) P<0.05,\left({ }^{* * *}\right) P<0.001$, for post hoc pairwise $t$-tests. (C) Time spent in a sleep-like state during the 17-h intervals between Training and Retrieval 1 and between Retrieval 1 and 2, for the Sleep (black bars) and Wake groups (white bars). (D) Correlation between time in a sleep-like state and performance change, in terms of total mouth time and total response time, across the Training-to-Retrieval 1 retention interval for the Sleep group, and $(E)$ across the Retrieval 1-to-2 retention interval for the Sleep and $(F)$ Wake group. Note, there were no significant correlations in the Wake group. Correlation analyses included animals that were otherwise excluded from the main analyses due to insufficient sleep after training (see Methods).

facilitating the accessibility of the memory. Nevertheless, and also in light of findings indicating that learning on the LFI task, due to circadian rhythms, might be limited to the active daytime period of the animals (Lyons et al. 2005), we performed an additional control experiment in which Retrieval 1 was omitted to further exclude retrieval-related confounds. The wake group of this experiment (WakeC, $n=17$ ) was kept awake the first $17 \mathrm{~h}$ after training (taking place in morning), then was allowed to sleep and retrieval was tested $\sim 34 \mathrm{~h}$ after training (Fig. 3). The sleep group (SleepC, $n=15$ ) was trained in the evening and retrieval was tested $\sim 34 \mathrm{~h}$ later, with normal sleep during the entire retention interval (Fig. 3A). Results confirmed a general improvement in LFI performance across the 34 -h retention interval expressed by a decrease in total mouth time $\left(F_{(1,30)}=83.65, P<0.0001\right.$, for Training/Retrieval main effect) which was significantly more pronounced in the SleepC than WakeC group $\left(F_{(1,30)}=10.25\right.$, $P<0.003$, for Training/Retrieval $\times$ SleepC/WakeC interaction, Fig. 3B). For total response time, the on average greater reduction in the SleepC than WakeC group failed to reach significance $\left(F_{(1,30)}=1.33, P=0.26\right.$ for Training $/$ Retrieval $\times$ SleepC $/$ WakeC, 
A

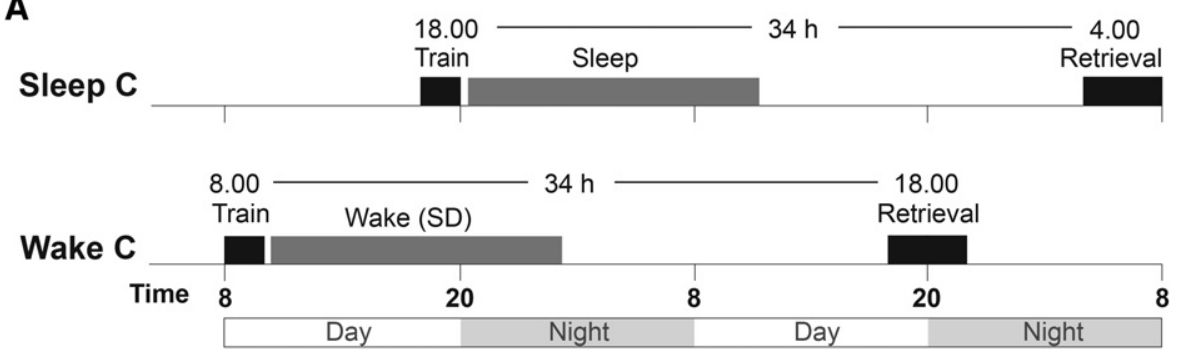

B
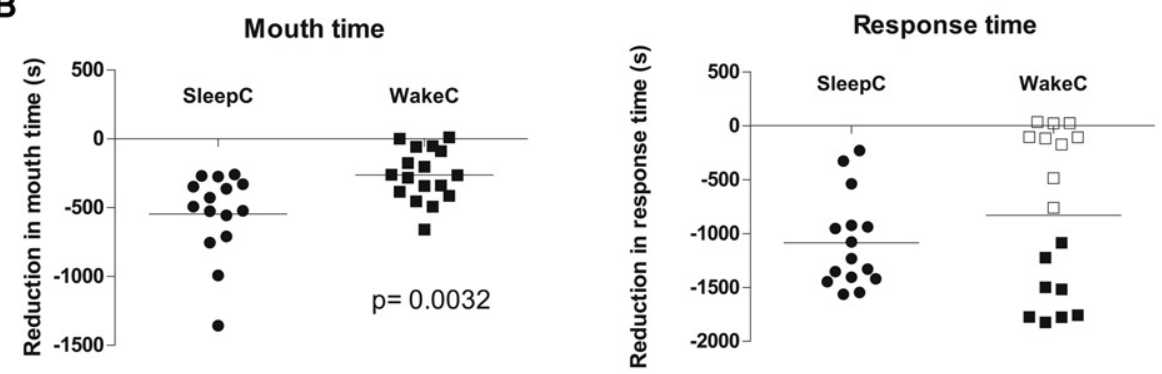

C

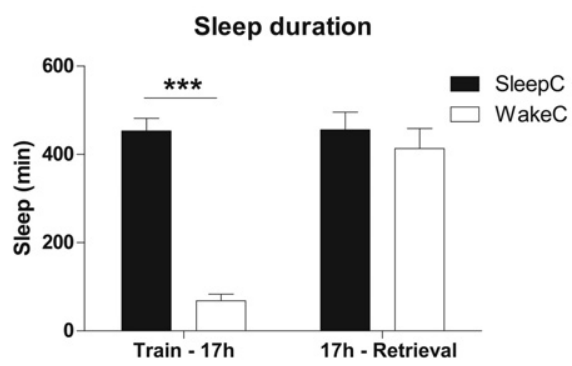

Figure 3. Design and results of control experiments. $(A)$ Design: A SleepC group $(n=15)$ and a WakeC group of animals $(n=17)$ were tested on two occasions, Training and Retrieval, separated by a 34-h retention interval. In the WakeC group Training was followed by a 17-h wake interval (supported by sleep deprivation if needed) whereas the animals of the Sleep group expressed spontaneous sleep in this interval. In the subsequent 17-h interval sleep was permitted in both groups. (B) (Left) Animals of the SleepC group show a stronger reduction in the mouth time from Training to Retrieval than animals of the WakeC group. (Right) Mean decreases in total response time from Training to Retrieval did not differ between groups $(P=0.258)$. Note that the WakeC group appears to split up in a subgroup with strong decreases (white boxes) and no decreases (filled boxes) in total response time (see text for further analyses. (C) Time spent in a sleep-like state during the first 17-h interval after Training and the subsequent second 17-h interval prior Retrieval for the SleepC (black bars) and WakeC groups (white bars). (**) $P<0.001$.

$F_{(1,30)}=75.17, P<0.0001$ for Training/Retrieval main effect). This appeared to be due to the distinctly larger variability in the reduction in total response time in the WakeC group including a subgroup of nine animals that, despite of the 17 -h posttraining wake interval, substantially reduced their total response time $\left(K^{2}=11.53, P=0.0031\right.$, D'Agostino $K^{2}$ test for deviation from normal distribution; $F=3.05, P=0.042$, for difference in variance between SleepC and WakeC groups, $t_{(15)}=10.33$, $P<0.0001$ for a post hoc comparison between the means of the subgroups, Fig. 3B).

Further exploratory comparisons indicated that these subgroups showing and not showing substantial decreases in total training time, respectively, did not differ with respect to their initial performance level during Training $\left(t_{(15)}=0.79, P=0.44\right)$. It might be that in the animals of the WakeC group showing distinct decreases in total response time, sleep occurring immediately after the 17-h wake period, rescued some residual traces from decay. Foregoing research suggested a wide time range (12-24 h after training) in which signs of long-term memory might develop (Sutton et al. 2001, Michel et al. 2012, 2013). Accordingly, the state of intermediate memory with a particular sensitivity to the effects of sleep might possibly last longer than $17 \mathrm{~h}$. Alternatively, it could be argued that sleep deprivation in the WakeC animals showing improvement across the retention interval was less effective. Our deprivation procedure allowed for short sleep bouts and in vertebrates, brief local sleep periods have been found to intrude wake, especially after longer periods of sleep deprivation (Vyazovskiy et al. 2011). Finally, it could also be argued that a primary effect of sleep is to reduce variance in the behavior regulated through the respective memory traces, which in the absence of sleep remain distinctly elevated.

Whatever the case, overall results from this control experiment support the notion that sleep in Aplysia benefits a post-training process of consolidation. Importantly, they simultaneously exclude that the worse performance of the Wake group in the main experiment at Retrieval 1 was exclusively owed to enhanced fatigue leading to an increased biting rate and a slower realization that the food is inedible. The design of the main experiment with a retention interval extending over $17 \mathrm{~h}$ avoided to a certain extent circadian confounds, since animals of the Wake group were trained in the morning, spending most of the day moving. Wakefulness had to be supported only during the evening hours. So the animal kept itself awake for most of the wake period, and the experimenter kept it artificially awake only toward the end of the 17- $h$ period. The Sleep group was trained in the late afternoon, just before going to sleep, and was tested $17 \mathrm{~h}$ later, in the late morning hours after having spent some hours in their active period. Even though Sleep and Wake groups of the main and control experiments were trained at different times of the day (8:00 $\mathrm{h}$ and 18:00 $\mathrm{h}$ ) initial training performance was the same and the majority of the animals of both groups showed clear signs of learning. This is consistent with previous studies that neither found a circadian acquisition nor recall effect for the two times of training used in our experiments (Fernandez et al. 2003; Lyons et al. 2005). Regarding the question whether the circadian night phase or sleep is the contributing factor for memory consolidation our experiments speak for sleep being more relevant to memory consolidation. This is further corroborated by the distinct correlation we found between sleep duration and LFI retrieval performance (Fig. 2E,F). The finding that even the animals that were kept awake showed significant memory at the later retrieval test suggests that rudimentary consolidation might also occur during wakefulness and that sleep, rather than initiating long-term memory consolidation, mainly acts to enhance such processes.

Our experiments add to previous studies in invertebrates indicating that sleep-like states that follow training support consolidation of memory for the respective task (Donlea et al. 2009, 2011; Hussaini et al. 2009; Zwaka et al. 2015; Levy et al. 2016), 
and, of note, they will complement a most recent study in Aplysia showing a similar suppression of memory for the LFI task after a shorter 9-h period of post-training sleep deprivation (Krishnan et al. 2016). Consolidating effects, in all these studies emerged in the absence of electrophysiological signs of sleep, as invertebrates have not been shown to display the characteristic electrophysiological signatures, such as slow waves, used to determine sleep in vertebrates. It has been hypothesized that only forms of learning that are more complex than classical conditioning and/or include an inhibitory component profit from sleep, as seen in Drosophila or bees (Vorster and Born 2015). This view is supported by the present findings, although we did not contrast effects of sleep on LFI with those on simpler tasks. LFI is indeed a complex multimodal form of conditioning in which the animal learns to inhibit its response toward a food stimulus. It, thus, shares similarities with extinction learning, which was found to be supported by sleep in bees (Hussaini et al. 2009).

Molecular changes in the expression of ApC/EBP after LFI training have been found to be localized in the buccal ganglia of Aplysia (Levitan et al. 2010). However, given the complexity of LFI, it most likely involves further ganglia, as it was shown for conditioned taste aversion in Lymnaea (Hatakeyama et al. 2006). Specifically, in the process of consolidation, molecular changes may arise at sites different from those involved in initial encoding of the information during training, similar to the systems consolidation process that has been proposed to occur during sleep in mammals. There, declarative memory representations residing in hippocampal network undergo reactivations during sleep that support a gradual redistribution of the representation toward neocortical networks serving as long-term store (Levitan et al. 2010; Vorster and Born 2015). In line with this, sleep-like states in Aplysia might enhance neuronal reorganization processes as they were revealed in Lymnaea, where neuronal reorganization accompanied the transition from intermediate to long-term memory without any apparent change in behavioral recall outcome (Braun and Lukowiak 2011). Notably, in bees the experimental reactivation of a memory by an odor cue presented during sleep enhanced a memory that was normally forgotten (Zwaka et al. 2015), which suggests that reorganization processes in invertebrates, as in mammals, originate from the reactivation of newly encoded representations during sleep. Ultimately, in this way the consolidation of memory for more complex behaviors during sleep might lead to a simpler neural representation, which contains only the adaptively relevant information, is more easily accessible, and requires less energy to be maintained.

\section{Acknowledgments}

We thank Frederik Weber for programming the software BitSeWe that was used to record the events during LFI training and testing. We thank Johanna Klees and Kathrin Thiede for assistance with animal maintenance and behavioral experiments. This research is supported by a grant from the DFG SFB 654 "Plasticity and Sleep."

\section{References}

Beyaert L, Greggers U, Menzel R. 2012. Honeybees consolidate navigation memory during sleep. J Exp Biol 215: 3981-3988.

Botzer D, Markovich S, Susswein AJ. 1998. Multiple memory processes following training that a food is inedible in Aplysia. Learn Mem 5: 204-219.

Braun MH, Lukowiak K. 2011. Intermediate and long-term memory are different at the neuronal level in Lymnaea stagnalis (L.). Neurobiol Learn Mem 96: 403-416.
Campbell SS, Tobler I. 1984. Animal sleep: a review of sleep duration across phylogeny. Neurosci Biobehav Rev 8: 269-300.

Cirelli C, Tononi G. 2008. Is sleep essential? PLoS Biol 6: e216.

Donlea JM, Ramanan N, Shaw PJ. 2009. Use-dependent plasticity in clock neurons regulates sleep need in Drosophila. Science 324: 105-108.

Donlea JM, Thimgan MS, Suzuki Y, Gottschalk L, Shaw PJ. 2011. Inducing sleep by remote control facilitates memory consolidation in Drosophila. Science 332: 1571-1576.

Fernandez RI, Lyons LC, Levenson J, Khabour O, Eskin A. 2003. Circadian modulation of long-term sensitization in Aplysia. Proc Natl Acad Sci 100: $14415-14420$.

Ganguly-Fitzgerald I, Donlea J, Shaw PJ. 2006. Waking experience affects sleep need in Drosophila. Science 313: 1775-1781.

Hatakeyama D, Sadamoto H, Watanabe T, Wagatsuma A, Kobayashi S, Fujito Y, Yamashita M, Sakakibara M, Kemenes G, Ito E. 2006. Requirement of new protein synthesis of a transcription factor for memory consolidation: paradoxical changes in mRNA and protein levels of C/EBP. J Mol Biol 356: 569-577.

Hussaini SA, Bogusch L, Landgraf T, Menzel R. 2009. Sleep deprivation affects extinction but not acquisition memory in honeybees. Learn Mem 16: 698-705.

Kandel ER, Dudai Y, Mayford MR. 2014. The molecular and systems biology of memory. Cell 157: 163-186.

Katzoff A, Ben-Gedalya T, Susswein AJ. 2002. Nitric oxide is necessary for multiple memory processes after learning that a food is inedible in Aplysia. J Neurosci 22: 9581-9594.

Krishnan HC, Gandour CE, Ramos JL, Wrinkle MC, Sanchez-Pacheco JJ, Lyons LC. 2016. Acute sleep deprivation blocks short and long-term operant memory in Aplysia. Sleep 39: 2161-2171.

Le Glou E, Seugnet L, Shaw PJ, Preat T, Goguel V. 2012. Circadian modulation of consolidated memory retrieval following sleep deprivation in Drosophila. Sleep 35: 1377-1384b.

Levitan D, Twitto R, Levy R, Lyons LC, Susswein AJ. 2010. A brief retraining regulates the persistence and lability of a long-term memory. Learn Mem 17: $402-406$.

Levitan D, Saada-Madar R, Teplinsky A, Susswein AJ. 2012. Localization of molecular correlates of memory consolidation to buccal ganglia mechanoafferent neurons after learning that food is inedible in Aplysia. Learn Mem 19: 503-512.

Levy R, Levitan D, Susswein AJ. 2016. New learning while consolidating memory during sleep is actively blocked by a protein synthesis dependent process. Elife 5: e17769.

Lyons LC, Rawashdeh O, Katzoff A, Susswein AJ, Eskin A. 2005. Circadian modulation of complex learning in diurnal and nocturnal Aplysia. Proc Natl Acad Sci 102: 12589-12594.

Michel M, Green CL, Lyons LC. 2011. PKA and PKC are required for long-term but not short-term in vivo operant memory in Aplysia. Learn Mem 18: 19-23.

Michel M, Green CL, Gardner JS, Organ CL, Lyons LC. 2012. Massed training-induced intermediate-term operant memory in Aplysia requires protein synthesis and multiple persistent kinase cascades. J Neurosci 32: 4581-4591.

Michel M, Gardner JS, Green CL, Organ CL, Lyons LC. 2013. Protein phosphatase-dependent circadian regulation of intermediate-term associative memory. J Neurosci 33: 4605-4613.

Sutton MA, Masters SE, Bagnall MW, Carew TJ. 2001. Molecular mechanisms underlying a unique intermediate phase of memory in Aplysia. Neuron 31: 143-154.

Vorster AP, Born J. 2015. Sleep and memory in mammals, birds and invertebrates. Neurosci Biobehav Rev 50: 103-119.

Vorster AP, Krishnan HC, Cirelli C, Lyons LC. 2014. Characterization of sleep in Aplysia californica. Sleep 37: 1453-1463.

Vyazovskiy VV, Olcese U, Hanlon EC, Nir Y, Cirelli C, Tononi G. 2011. Local sleep in awake rats. Nature 472: 443-447.

Watson BO, Buzsáki G. 2015. Sleep, memory \& brain rhythms. Daedalus 144: $67-82$.

Westermann J, Lange T, Textor J, Born J. 2015. System consolidation during sleep - a common principle underlying psychological and immunological memory formation. Trends Neurosci 38: 585-597.

Zwaka H, Bartels R, Gora J, Franck V, Culo A, Götsch M, Menzel R. 2015. Context odor presentation during sleep enhances memory in honeybees. Curr Biol 25: 2869-2874.

Received January 19, 2017; accepted in revised form March 19, 2017. 


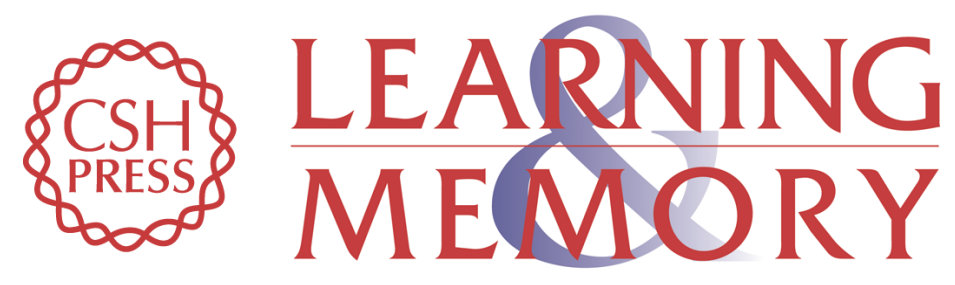

\section{Sleep supports inhibitory operant conditioning memory in Aplysia}

Albrecht P. A. Vorster and Jan Born

Learn. Mem. 2017, 24:

Access the most recent version at doi:10.1101/Im.045054.117

References This article cites 29 articles, 13 of which can be accessed free at: http://learnmem.cshlp.org/content/24/6/252.full.html\#ref-list-1

Creative This article, published in Learning \& Memory, is available under a Creative Commons Commons License (Attribution-NonCommercial 4.0 International), as described at License http://creativecommons.org/licenses/by-nc/4.0/.

Email Alerting Receive free email alerts when new articles cite this article - sign up in the box at the Service top right corner of the article or click here. 